54, 1, pp. 149-161, Warsaw 2016

DOI: $10.15632 /$ jtam-pl.54.1.149

\title{
MODELLING AND SIMULATION STUDIES ON THE MOBILE ROBOT WITH SELF-LEVELING CHASSIS
}

\author{
JACEK BAŁCHANOWSKI \\ Wroctaw University of Technology, Faculty of Mechanical Engineering, Wroctaw, Poland \\ e-mail: jacek.balchanowski@pwr.edu.pl
}

\begin{abstract}
The mobile robot presented in the article is a hybrid system combining efficient travel on wheels on a flat terrain with the capability of surmounting obstacles by walking. The research is focused on designing a control system maintaining the robot chassis at a constant position to the ground. The aims of this research are: creation of the computational model of the control system for the levelling system of designed mobile robots and realization of simulation studies on the robots travel in terrain with obstacles. The simulations aimed at determination of basic dynamic and kinematic properties.
\end{abstract}

Keywords: mobile robot, simulation studies, self-leveling chassis

\section{Introduction}

The research on mobile robots has intensified in the recent years, especially to meet the demand for automating the transport process and for inspection (chemically, biologically) of contaminated areas and those exposed to hazard of fire explosions (Tchoń et al., 2000; Trojnacki et al., 2008).

Studies focused on mobile robots have been carried out in many research centers (universities, military and industrial centers). They deal with wheeled robots, walking robots, tracking robots, crawling robots, flying robots, floating robots and their hybrids. The research has been calculated on variety of such vehicles differing in their way of traveling: wheeled systems (WalkPartner, see Halme et al., 2003), tracked systems (INSPECTOR Robot, see Hołdanowicz, 2008), walking systems (PetMan, see Boston Dynamic, 2014), floating and flying systems (Hermes ${ }^{\circledR}$ 900, see Elbit Systems, 2014).

The dominant contemporary form of vehicles motion is riding on wheels. In an urban area, where the surface is smooth, the wheels are the most effective. However, the biggest disadvantage is that they have no ability to overcome obstacles in form of a substrate discontinuity - curbs, stairs, slopes. The most common form of motion by living organisms of the Earth is treading. This type of transportation is especially effective with moving around non-urbanized irregular surfaces containing obstacles (Bałchanowski and Gronowicz, 2012a,b; Zielińska,2003).

Mobile wheel-legged robots are hybrids that combine efficient travelling on a flat terrain by wheels with the capability of surmounting obstacles by walking. A major challenge in designing such systems is to develop its wheel suspension allowing the robot both to move on wheels and to walk, and automatically level its chassis during travelling on an uneven surface (Bałchanowski and Gronowicz, 2012a,b; Gronowicz and Szrek, 2009a,b; Szrek and Wójtowicz, 2010).

One such system is a wheel-legged mobile robot (Fig. 1) designed and built at Wroclaw University of Technology (Bałchanowski, 2012; Bałchanowski and Gronowicz, 2012a,b). The robot is equipped with a unique wheel suspension which allows it to drive, walk, rise, lower and self-level the chassis. In this paper, the design of this device is described. 

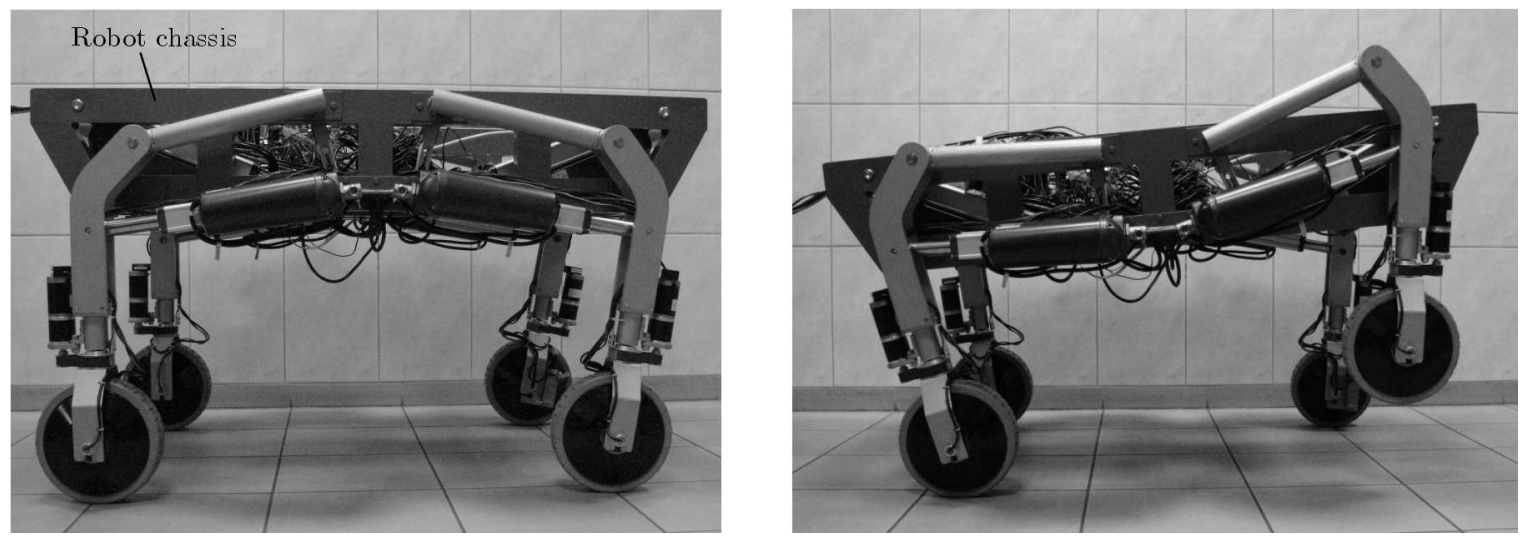

Fig. 1. A general view of a mobile robot and a view of the walking phase

\section{Design of a mobile robot}

In the framework of the project realized at Wroclaw University of Technology the design of a robot whose schematic is shown in Fig. 2 has been developed. It is assumed that the wheels with suspensions are symmetrically arranged in relation to the longitudinal and transverse axis of the robot. Such a position of the wheels ensures a level playing field for driving of the front and rear axles. The major design challenge was to develop a suspension mechanism which should provide the robot with ability to walk with a view system to overcome obstacles on the track and enabling automatic self-levelling of the chassis (Fig. 3).

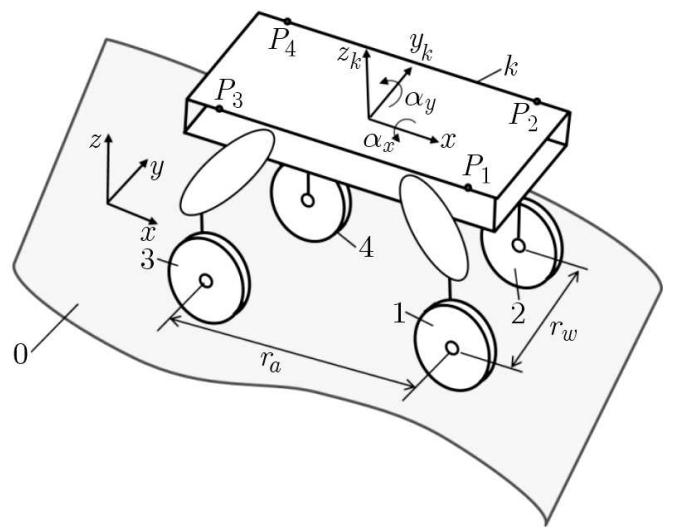

Fig. 2. A general scheme of the wheel-legged mobile robot (1-4- wheel suspensions, 0 - ground, $k$ - chasis)

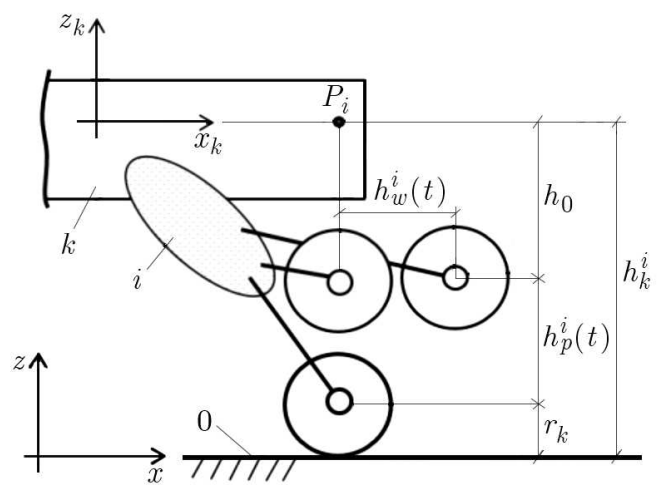

Fig. 3. A schematic showing the execution of suspension $i$ motions: $h_{p}^{i}-\operatorname{lifting}, h_{w}^{i}-$ ejecting 
The wheel suspension is a complex mechanism with 4 degrees of freedom in relation to the body. Such a mechanism (Fig. 3) must ensure the full range of motion in order to fulfill the task of driving (turning and twisting wheels - two degrees of freedom) as well as lifting and ejecting the wheels (two degrees of freedom).

As a result of the design work on the suspension structure and then on the geometric synthesis, main dimensions of system have been chosen (Bałchanowski, 2012; Bałchanowski and Gronowicz, 2012c; Gronowicz et al., 2012; Sperzyński et al., 2010; Szrek and Wójtowicz, 2010). A view of the right front side of the robot with wheel suspension mechanism 1 robot is given in Fig. 4. For a single suspension, the lifting of the wheel $h_{p}^{i}$ is realized by a linear actuator $q_{p}^{i}$. The ejecting of the wheel $h_{w}^{i}$ is by means of linear actuators $q_{w}^{i}$.

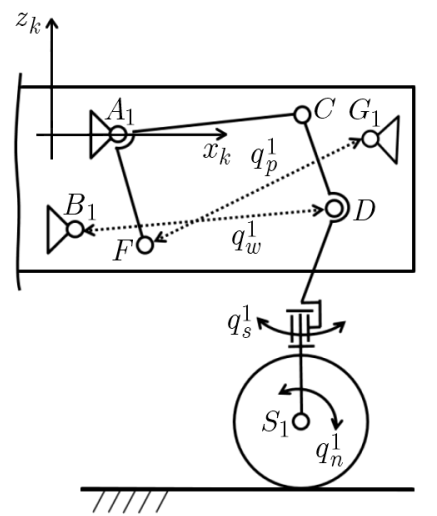

Fig. 4. The kinematic scheme of mobile robot suspension (front right side)

The robot is designed for inspection work both outdoors and indoors (e.g. buildings, production halls, etc.). Since it is designed to move inside rooms, to pass through typical doorways (less than $0.9 \mathrm{~m}$ wide) and to be able to surmount an obstacle with a height equal to that of a typical stair step (the wheel lifting height greater than $0.2 \mathrm{~m}$ ), its overall dimensions have to be limited. The chosen axle base $r_{a}$ is $0.8 \mathrm{~m}$ and the wheels base $r_{w}$ is $0.65 \mathrm{~m}$ (Fig. 2). The rest of basic parameters of geometric wheel suspension 1 is shown in Table 1.

Table 1. Geometric parameters of wheel suspension 1

\begin{tabular}{|c|c|c|c|c|c|}
\hline Parameter & Value & Parameter & Value & Parameter & Value \\
\hline \hline$x_{A_{1}}$ & $0.11 \mathrm{~m}$ & $y_{A_{1}}$ & $-0.65 \mathrm{~m}$ & $z_{A_{1}}$ & $0 \mathrm{~m}$ \\
\hline$x_{B_{1}}$ & $-0.04 \mathrm{~m}$ & $y_{B_{1}}$ & $-0.65 \mathrm{~m}$ & $z_{B_{1}}$ & $-0.152 \mathrm{~m}$ \\
\hline$x_{G_{1}}$ & $0.518 \mathrm{~m}$ & $y_{G_{1}}$ & $-0.65 \mathrm{~m}$ & $z_{G_{1}}$ & $0.005 \mathrm{~m}$ \\
\hline$D S_{1}$ & $0.253 \mathrm{~m}$ & $A_{1} F$ & $0.17 \mathrm{~m}$ & $A_{1} C$ & $0.303 \mathrm{~m}$ \\
\hline$C S_{1}$ & $0.5 \mathrm{~m}$ & $C D$ & $0.162 \mathrm{~m}$ & $h_{0}$ & $0.335 \mathrm{~m}$ \\
\hline
\end{tabular}

The lifting and ejection can be achieved with linear drives, e.g. electric actuators LINAK LA36. Solid rubber-steel wheels with a motor and a gear integrated with a hub (GOLDENMOTOR HUB24E) have been chosen as the travelling drives (Bałchanowski, 2012; Bałchanowski and Gronowicz, 2012a,b). The main specifications of the wheel drives as well as the lift and ejection-protrusion actuators are shown in Table 2.

On the basis of the developed conceptual design and documentation, a prototype of a mobile wheel-legged robot has been made (Fig. 1).

When driving on uneven ground, the robot chassis is rotated along the longitudinal and transverse axes. The implementation of the levelling aims to maintain a constant orientation of 
Table 2. Main parameters of the drives

\begin{tabular}{|c|c|c|}
\hline \multicolumn{3}{|c|}{ Actuator LINAK LA36 } \\
\hline \hline \multirow{3}{*}{} & $q_{w}, q_{p}$ (stroke length) & $0.35-0.5 \mathrm{~m}$ \\
\cline { 2 - 3 } & $v_{w}, v_{p}$ (speed) & $0.068 \mathrm{~m} / \mathrm{s}$ \\
\cline { 2 - 3 } & $F_{w}, F_{p}$ (force) & $1700 \mathrm{~N}$ \\
\cline { 2 - 3 } & $m_{s}$ (mass) & $4.9 \mathrm{~kg}$ \\
\hline \hline \multirow{3}{*}{} & Wheel GOLDENMOTOR HUB24 \\
\hline \multirow{3}{*}{} & $d q_{n} / d t$ (angular velocity) & $13.08 \mathrm{rad} / \mathrm{s}(125 \mathrm{rpm})$ \\
\cline { 2 - 3 } & $M_{n}$ (nominal torque) & $13.5 \mathrm{Nm}$ \\
\cline { 2 - 3 } & $k_{r}$ (radial stiffness) & $9.5 \cdot 10^{5} \mathrm{~N} / \mathrm{m}$ \\
\cline { 2 - 3 } & $m_{k}$ (mass) & $5 \mathrm{~kg}$ \\
\cline { 2 - 3 } & $r_{k}$ (radius of wheel) & $0.105 \mathrm{~m}$ \\
\hline
\end{tabular}

the robot body above the ground according to the scheme shown in Figs. 2 and 3, which means maintaining the value of the given angles of orientation

$$
\alpha_{x}=0 \quad \alpha_{y}=0
$$

Raising or lowering the individual wheels can bring the robot to the assumed level. This function can be accomplished solely by lifting the chassis by means of the lifting actuators $q_{p}^{i}$ (Fig. 4), while the other drives (ejection, turn and rolling) remain fixed.

For the given values of wheels radii $r_{k}$ and suspension height $h_{0}$, the height $h_{k}^{i}$ of the robot chassis above the ground may be presented in the form (Fig. 3)

$$
h_{k}^{i}=r_{k}+h_{0}+h_{p}^{i}\left(q_{p}^{i}\right)
$$

The graph in Fig. 5 shows changes of the height $h_{p}^{i}$ for the suspension as a function of the actuator extension $q_{p}^{i}$ (Bałchanowski and Gronowicz, 2012a,b,c). For the adopted actuator stroke $q_{p}^{i}=0.35-0.5 \mathrm{~m}$ (Table 2), the defined range of changes of the wheel lifting $h_{p}^{i}$ is

$$
0 \leqslant h_{p}^{i} \leqslant 0.26 \mathrm{~m}=h_{p}
$$

where $h_{p}$ is the maximum height of the suspension lifting.

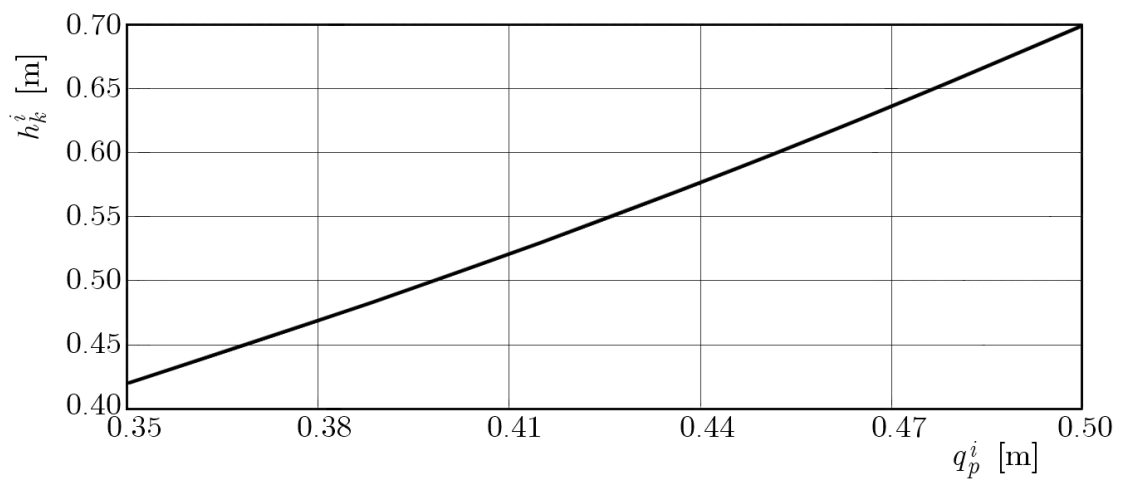

Fig. 5. Elevation of the robot chassis $h_{k}^{i}$ versus extension of the lifting actuator $q_{p}^{i}$

The maximum value of the wheel lifting height $h_{p}$ determines the possibility of overcoming a certain unevenness. The mechanism shown in side and front views on uneven ground is presented in Fig. 6. The maximum angles of the ground inclination $\alpha_{x}^{\max }$ along the robot longitudinal $x$ axis as well as $\alpha_{y}^{\max }$ along the robot transverse $y$ axis, can be determined from the relationship

$$
\alpha_{x}^{\max }=\arctan \frac{h_{p}}{r_{w}}=21.8^{\circ} \quad \alpha_{y}^{\max }=\arctan \frac{h_{p}}{r_{a}}=18.0^{\circ}
$$



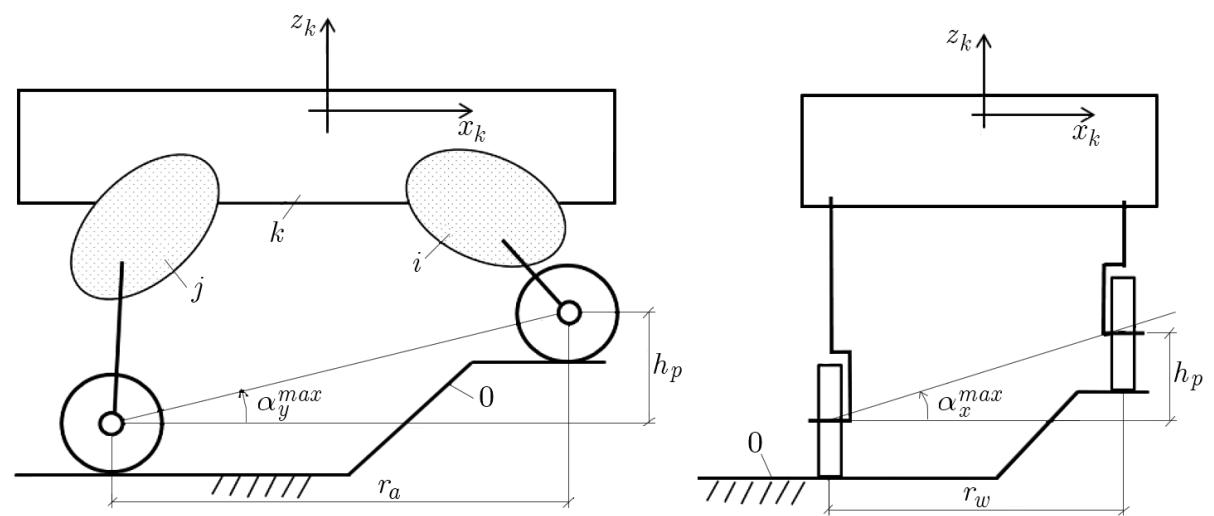

Fig. 6. Side and front views of the robot on uneven ground. The schematic shows the maximum angles of the ground inclination $\alpha_{x}^{\max }$ and $\alpha_{y}^{\max }$ along the robot longitudinal $x$ and transverse $y$ axis

If the area has larger values of the inclination angles, then the lifting mechanisms do not provide sufficient levelling of the chassis.

\section{Numerical model of the mobile robot}

In order to perform simulations, a computational model of the wheel-legged robot shown in Fig. 7, has been created in the LMS DADS (Haug, 1989) dynamic analysis system. The robot has $22 \mathrm{DOF}$, with the body having 6 DOF and each wheel suspension having 4 DOF relative to the body. Sixteen kinematic excitations are defined in the robot: 8 rotational excitations $q_{n}^{i}$ and $q_{s}^{i}$ (wheel rolling and turning) as well as 8 linear excitations $q_{p}^{i}$ and $q_{w}^{i}$ (wheel lifting and ejecting) for each suspension $(i=1,2,3,4)$ (Bałchanowski, 2012; Bałchanowski and Gronowicz, 2012a,b).

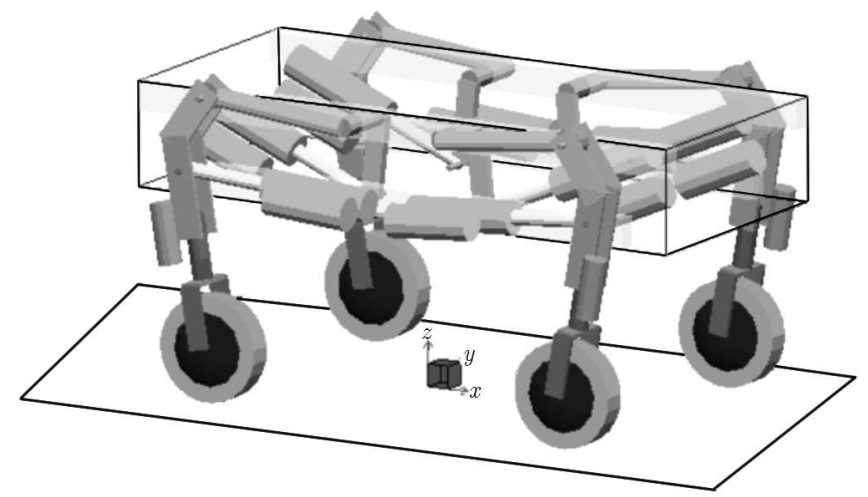

Fig. 7. The model of the wheel-legged mobile robot (main view)

The wheel/base interactions are modelled using a tire/ground interaction force model (TIRE) (Haug, 1989). The mass of the wheels is quite large due to the fact that the motor and gear are incorporated in the hub, and because of their high radial and longitudinal stiffness (Table 2).

The total weight (deadweight + payload) of the wheeled-legged robot is estimated at $100 \mathrm{~kg}$. The mass and geometry of the suspension, wheel and actuator parts are assumed as in the design. The weight of the body (comprising deadweight of the frame bearer, steering system, batteries, current generator as well as payload) is appropriately matched to obtain the assumed total weight of $100 \mathrm{~kg}$, with the center of gravity located in the body center. 


\subsection{Design of the control system for the levelling mechanism of the robot chassis}

When the robot travels on an uneven substrate, the robot chassis changes its orientation relative to the ground. The changes in orientation of the chassis are described by the angles of inclination $\alpha_{x}$ (the angle of the body rotation relative to the robot transverse axis) and the steering angle $\alpha_{y}$ (the angle of the body rotation relative to the robot longitudinal axis, Fig. 2). In a real robot, both angles (Fig. 1) are measured using inclinometers (Bałchanowski and Gronowicz, 2012b; Gronowicz and Szrek, 2009a,b; Szrek and Wójtowicz, 2010).

The plane of the robot chassis will be twisted as a result of rotations $\alpha_{x}$ and $\alpha_{y}$. The twisting can be described by means of $h_{1}, h_{2}, h_{3}$ and $h_{4}$ vertical displacements of points $P_{1}, P_{2}, P_{3}$ and $P_{4}$ (Figs. 2 and 8). For angles $\alpha_{x}, \alpha_{y}$, the position of points $P_{1}$ in the global coordinate system $x y z$, described by the vector $\mathbf{r}_{P_{i}}=\left[x_{P_{i}}, y_{P_{i}}, z_{P_{i}}\right]^{\mathrm{T}}$, can be calculated using the following formula

$$
\mathbf{r}_{P_{i}}=\mathbf{A}_{x} \mathbf{A}_{y}{ }^{k} \mathbf{r}_{P_{i}}
$$

where $i$ is the number of suspension, $i=1, \ldots, 4, \mathbf{A}_{x}$ - matrices of rotation from the $k$-th system to the $x y z$ system about the angle $\alpha_{x}$ along the $x^{6}$ axis, $\mathbf{A}_{y}$ - matrices of rotation from the $k$-th system to the $x y z$ system about the angle $\alpha_{y}$ along the $y$ axis

$$
\mathbf{A}_{x}=\left[\begin{array}{cccc}
1 & 0 & 0 & 0 \\
0 & \cos \alpha_{x} & -\sin \alpha_{x} & 0 \\
0 & \sin \alpha_{x} & \cos \alpha_{x} & 0 \\
0 & 0 & 0 & 1
\end{array}\right] \quad \mathbf{A}_{y}=\left[\begin{array}{cccc}
\cos \alpha_{y} & 0 & \sin \alpha_{y} & 0 \\
0 & 1 & 0 & 0 \\
-\sin \alpha_{y} & 0 & \cos \alpha_{y} & 0 \\
0 & 0 & 0 & 1
\end{array}\right]
$$

and ${ }^{k} \mathbf{r}_{P_{i}}$ - position vector of point $P_{i}$ on the chassis in the $x_{k} y_{k} z_{k}$ coordinate system

$$
\begin{array}{ll}
k_{\mathbf{r}_{P_{1}}}=\left[r_{a} / 2, r_{w} / 2,0,1\right]^{\mathrm{T}} & k_{\mathbf{r}_{P_{2}}}=\left[r_{a} / 2,-r_{w} / 2,0,1\right]^{\mathrm{T}} \\
k_{\mathbf{r}_{P_{3}}}=\left[-r_{a} / 2, r_{w} / 2,0,1\right]^{\mathrm{T}} & k_{\mathbf{r}_{P_{4}}}=\left[-r_{a} / 2,-r_{w} / 2,0,1\right]^{\mathrm{T}}
\end{array}
$$

Finally, the value of $h_{i}$ is described by the $z_{P_{i}}$ coordinate of the vector $\mathbf{r}_{P_{i}}$ from formula (3.1)

$$
h_{i}=z_{P_{i}} \quad i=1, \ldots, 4
$$

In order to bring the robot chassis plane to the level, the points $P_{1}, P_{2}, P_{3}$ and $P_{4}$ need to be moved to the designated values of $h_{1}, h_{2}, h_{3}, h_{4}$. The displacements $h_{i}$ are the disruptions for the leveling control system of the robot chassis. The control system has to set the proper wheel elevation $h_{i}$ using the linear actuators $q_{p}^{i}$ to bring the robot chassis to the level $\left(\alpha_{x}=0\right.$ and $\left.\alpha_{y}=0\right)$.

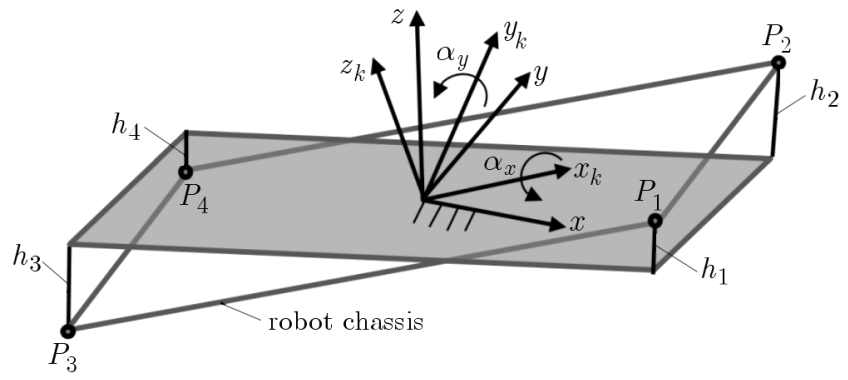

Fig. 8. The scheme of the robot chassis orientation angles

This requires controlling of only wheel lifting drives $q_{p}^{i}$ i.e., forcing the suspension displacement of $q_{p}^{i}$ by using forces $F_{p}^{i}$ from the actuators.

For a mobile robot on four wheels equipped with mechanisms for raising and lowering, the all-wheel task of setting a specific orientation of the chassis for uneven ground can be realized in 
many ways (Fig. 9) for different settings of the wheel height $h_{k}^{i}$ in the permissible range of the stroke $h_{p}$. In the proposed algorithm of automatic positioning and orientation of the chassis, in order to obtain one solution, it is assumed that the suspensions of three wheels are active and the forth one is the leading wheel with a predetermined height $h_{l}$ (Fig. 10).
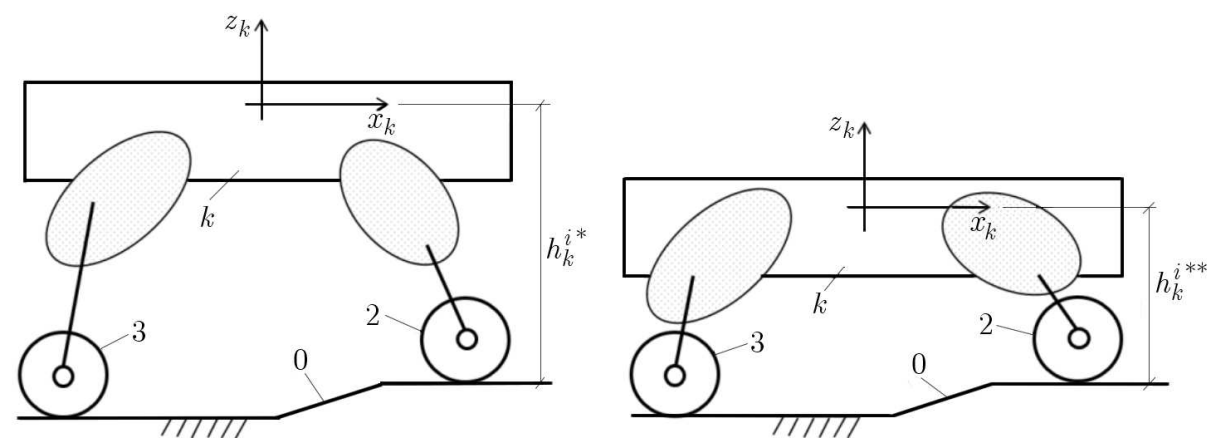

Fig. 9. A schematic showing examples of robot positions on uneven ground for different settings of the wheel heights $h_{k}^{i *}$ and $h_{k}^{i * *}$
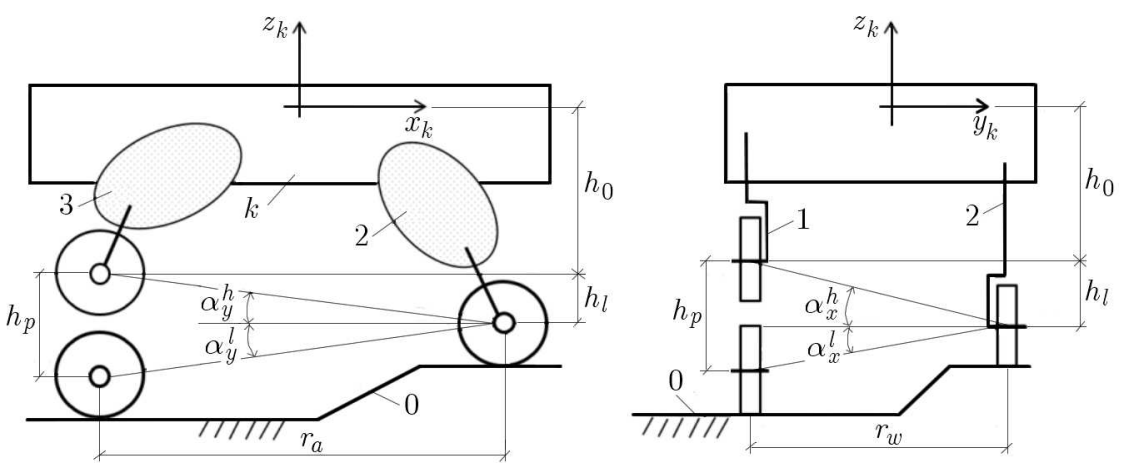

Fig. 10. The robot on the uneven ground with leading wheel 2 in side and front views. A schematic showing the maximum angles of ground inclination $\alpha_{x}^{h}, \alpha_{x}^{l}$ and $\alpha_{y}^{h}, \alpha_{y}^{l}$

In the work, it is assumed that the leading wheel is wheel 2 (left front). For such a proposed method of levelling, only one solution of searched heights $h_{i}$ will always be obtained for a given position of the body. The height $h_{l}$ of the leading wheel can be set in the range of

$$
0 \leqslant h_{l} \leqslant h_{p}
$$

For the adopted height $h_{l}$, the leading wheel possible changes in the orientation angles can be determined by formulas (Fig. 10)

$$
\begin{array}{rlrl}
\alpha_{y}^{h} & =\arctan \frac{h_{l}}{r_{a}} & \alpha_{y}^{l} & =\arctan \frac{h_{p}-h_{l}}{r_{a}} \\
\alpha_{x}^{h}=\arctan \frac{h_{l}}{r_{w}} & \alpha_{x}^{l}=\arctan \frac{h_{p}-h_{l}}{r_{w}}
\end{array}
$$

The height $h_{l}$ can be dynamically determined depending on the nature of the mobile robot ride and the existing uneven ground. In driving the robot on grounds with a positive angle (uphill), in order to increase the possibility of levelling the body, $h_{l}$ should have values close to zero in order to get the angle $\alpha_{y}^{l}$ according to (3.4), reaching its maximum value.

When driving the robot on the ground with a negative angle (down), $h_{l}$ value should be close to $h_{p}$ to obtain the angle $\alpha_{y}^{h}$ reaching the maximum value. When driving in the area with an undetermined uneven ground, $h_{l}$ should have a value of $h_{p} / 2$. 
As a result of the control model with leading wheel 2 (front left) having the fixed height $h_{l}$ while levelling displacement of the body, the values $h_{i}$ should be corrected about the value $h_{l}$

$$
h_{1}^{c}=h_{1}-h_{l} \quad h_{3}^{c}=h_{3}-h_{l} \quad h_{4}^{c}=h_{4}-h_{l}
$$

The corrected values $h_{i}^{c}$ will be disruptions to the regulators which control the raising of active wheel 1, 3 and 4 (front right, rear left and right). The regulators of the active wheels will reset the disruption $h_{i}^{c}$ to zero. In the structure of the levelling algorithm, there are three active regulators that control the raising and lowering of the active wheels 1, 3 and 4. In Fig. 11, a block algorithm of the platform levelling system of the robot chassis is shown.

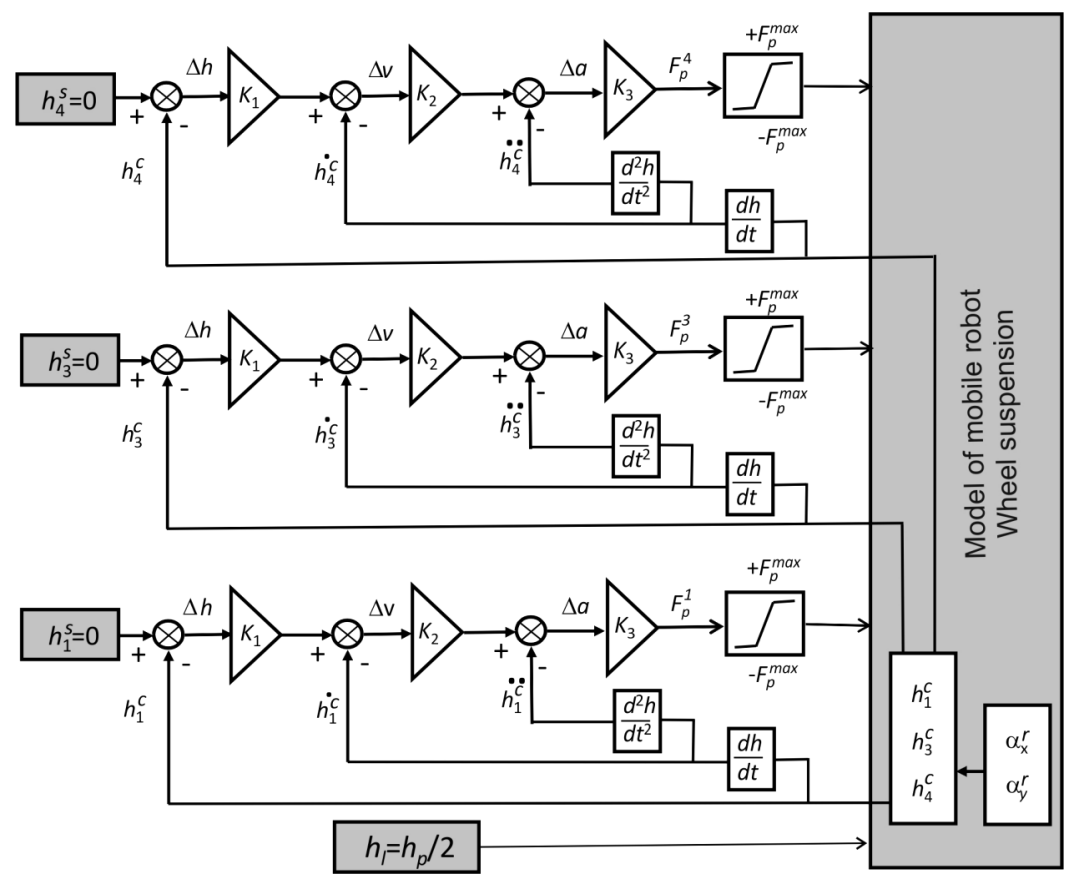

Fig. 11. A general diagram of the levelling control system

The inclinometers mounted on the robot body measure the distortions in form of orientation angles $\alpha_{x}^{r}, \alpha_{y}^{r}$ of twisting of the chassis while driving. These values will be used for calculation from formula (3.5) the displacement $h_{i}^{c}$ needed to bring the chassis to the level. The output of the regulator wheel is the force $F_{p}^{i}$ which causes the displacement $q_{p}^{i}$ of the actuator which controls raising and lowering of the active wheel $i$. The proposed control system has a closed structure with three feedback loops controlling the elevation $h_{k}^{i}$. The regulators control the actuators $q_{p}^{i}$ by determination of the active force $F_{p}^{i}$. The heights $h_{1}^{c}, h_{3}^{c}, h_{4}^{c}$ of the active wheels $1,3,4$ relative to the chassis will be controlled in closed loops.

In this control system, an external control loop computes the difference between the prescribed robot chassis elevation $h_{i}^{s}\left(h_{i}^{s}=0\right.$ in the case of levelling) and the actual chassis elevation $h_{i}^{c}$ calculated on the basis of the angles $\alpha_{x}^{r}, \alpha_{y}^{r}$ read from the chassis location. The computed elevation deviation $\Delta h$ passes through proportional controllers with constants $K_{1}, K_{2}, K_{3}$, generating a signal specifying the required demand for the active lifting force $F_{p}^{i}$, which is applied to the driving link of the robot. The control system incorporates blocks limiting the generated value of the force $F_{p}^{i}$ to the maximum values $\left(-F_{p}^{\max }<F_{p}<F_{p}^{\max }=1700 \mathrm{~N}\right)$ which the lifting actuator is capable of generating. Besides the robot, a computational model of the designed control system has been created in LMS DADS in order to study its dynamics.

The control parameters, i.e. constants $K_{1}, K_{2}$ and $K_{3}$ of the controllers need to be defined and matched. The parameters depend on the character of the object (the controlled mechanism). In 
control theory, there are many methods of matching such parameters. In this work, a numerical parameter matching procedure based on the Ziegler-Nichols method has been carried out. The simulations have been run in LMS DADS. The results of the controller parameter matching are: $K_{1}=600, K_{2}=100$ and $K_{3}=100$.

\section{Simulation examinations of the mobile robot with a leveling control system}

In order to determine dynamical properties of the mechanism and to verify the control system matching, motion of the system on an uneven surface has been simulated. A schematic of the simulation is shown in Fig. 12. The surface bumps are built of wedge-shaped obstacle drive-ons and drive-offs. The variation in the route height along the direction of motion for left and right side wheels is shown in Fig. 13.

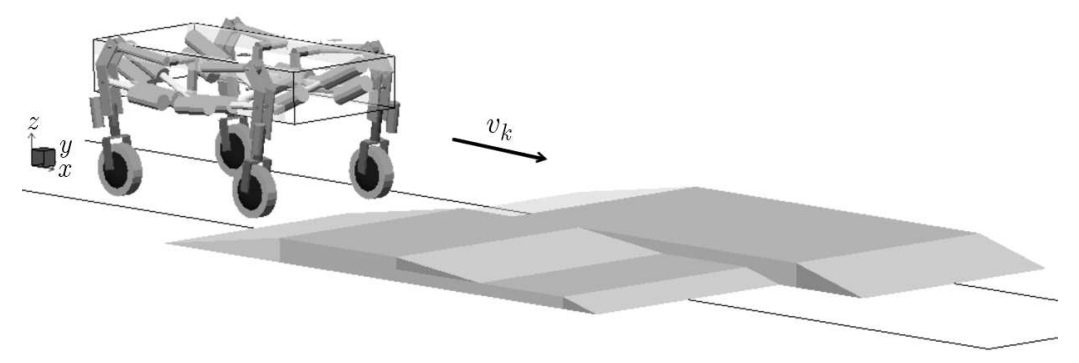

Fig. 12. The model of the mobile robot and a general scheme of simulation

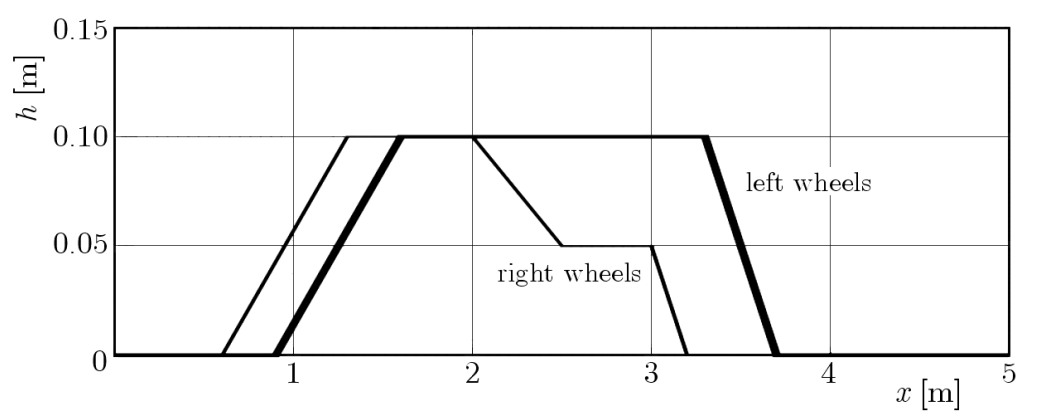

Fig. 13. Variation of heights $h$ of the uneven ground under the left and right wheel along the axis of motion

The parameters of the control system are orientation angles $\alpha_{x}=\alpha_{y}=0$ of the robot chassis with respect to the ground. They are constant during movement. It is assumed that the system would move at constant speed $v_{k}=1.0 \mathrm{~m} / \mathrm{s}(3.6 \mathrm{~km} / \mathrm{h})$. Wheel 2 is adopted as the leading wheel with the height $h_{l}=h_{p} / 2$. The terrain uneveness does not exceed the range of possible changes in the orientation angles $\alpha_{x}^{h}, \alpha_{x}^{l}, \alpha_{y}^{h}$ and $\alpha_{y}^{l}$ defined by formula (3.4). It is expected that during driving, the robot chassis will be kept at a given level. One of the aims of the simulations is to determine the control system response for the adopted excitations of motion. In particular, the accuracy of setting the orientations $\alpha_{x}, \alpha_{y}$ and the determination of active forces $F_{p}^{i}$ in the actuators $q_{p}^{i}$ have been ensured and executed.

The diagrams in Figs. 14 to 20 show the results of simulations in LMS DADS. Figures 14 and 15 show the variation in the real elevation $h_{k}^{i}$ of the robot chassis (coordinates $z$ of points $P_{i}$ - Fig. 2 and 8) and the trajectories of the centres $z_{s}^{i}$ of wheels 1, 2, 3 and 4 .

The quality of the control is illustrated in the next diagram where the errors $\alpha_{x}$ and $\alpha_{y}$ in the execution of the chassis levelling are shown (Fig. 16). The accuracy of the chassis orientation angles $\alpha_{x}$ and $\alpha_{y}$ below $0.5 \mathrm{deg}$ has been achieved. The control system quickly reacts to 


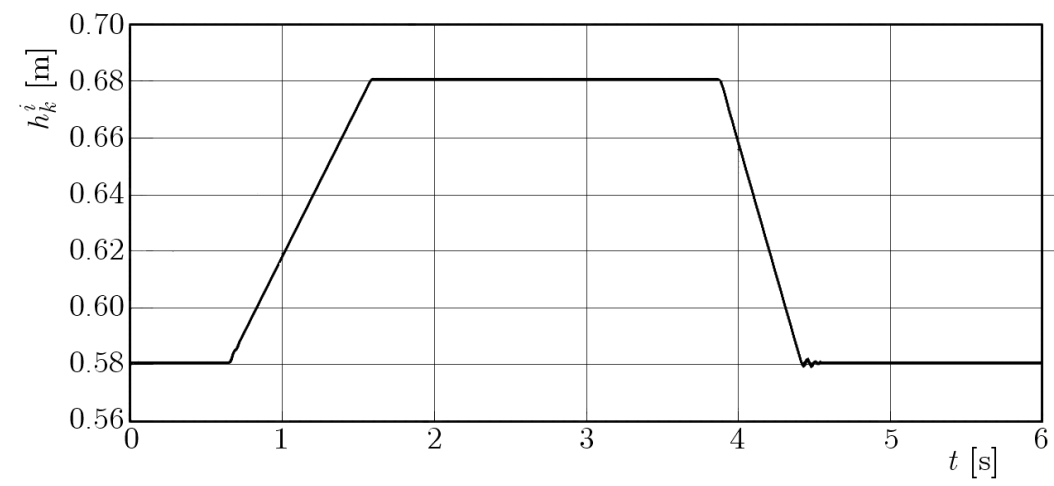

Fig. 14. Variation of the real elevations $h_{k}^{i}$ of the robot chassis during movement

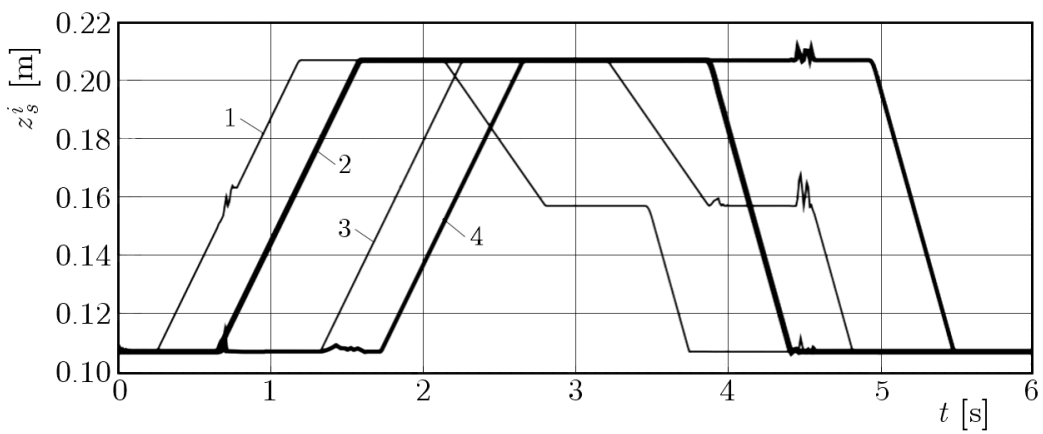

Fig. 15. Displacements of the centers $z_{s}^{i}$ of wheels 1, 2, 3 and 4 during movement

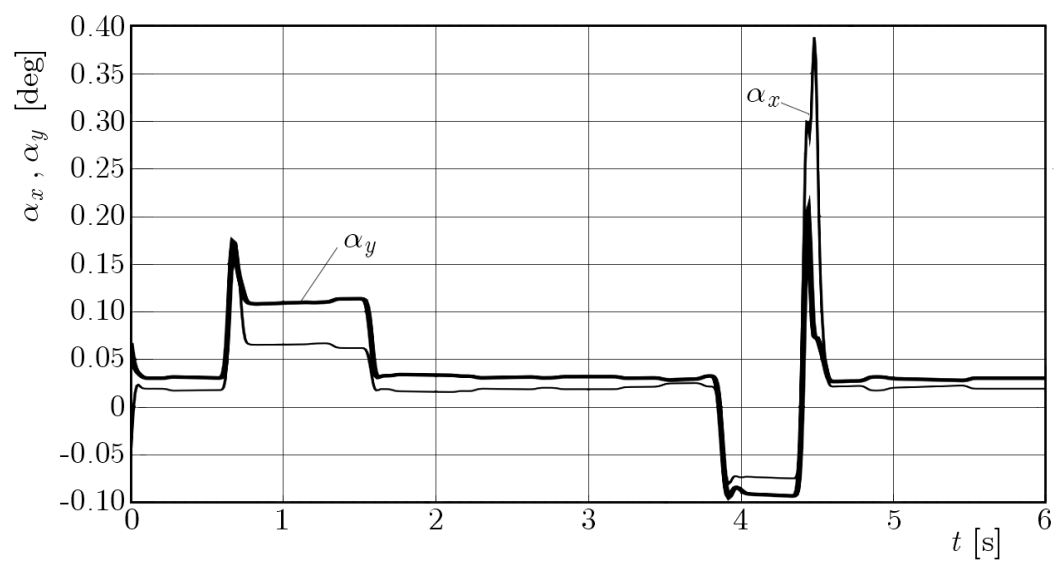

Fig. 16. Angles of orientation $\alpha_{x}, \alpha_{y}$ of the robot chassis found from simulations

disturbances in the surface bumps (points for $t=0.3,0.6,1.4,1.7,2.6,3.4,4.4,4.8,4.9,5.1 \mathrm{~s}$ in figures). The control system handles well the uneven ground, quickly stabilizing the robot.

The next figures show parameters of the lifting actuator. Figures 17 and 18 show the variation in length $q_{p}^{i}$ and velocity $v_{p}^{i}$ of the lifting actuator while Fig. 19 shows diagrams of the computed active force $F_{p}^{i}$ in these actuators. The wheel-ground interaction forces $F_{k}^{i}$ $(i=1,2,3,4)$ are presented in Fig. 20.

The analysis of the levelling system reveals that its performance of the latter mainly depends on height of the obstacle and robot travelling speed $v_{k}$. These quantities determine the vertical velocity component $z$ of the wheel which must be cancelled out by the opposite vertical motion of the chassis effected by the lifting actuator $q_{p}^{i}$ moving with an appropriate velocity $v_{p}^{i}$ (Fig. 17) and generating an appropriate active force $F_{p}^{i}$ (Fig. 19). The choice of a proper lifting actuator 


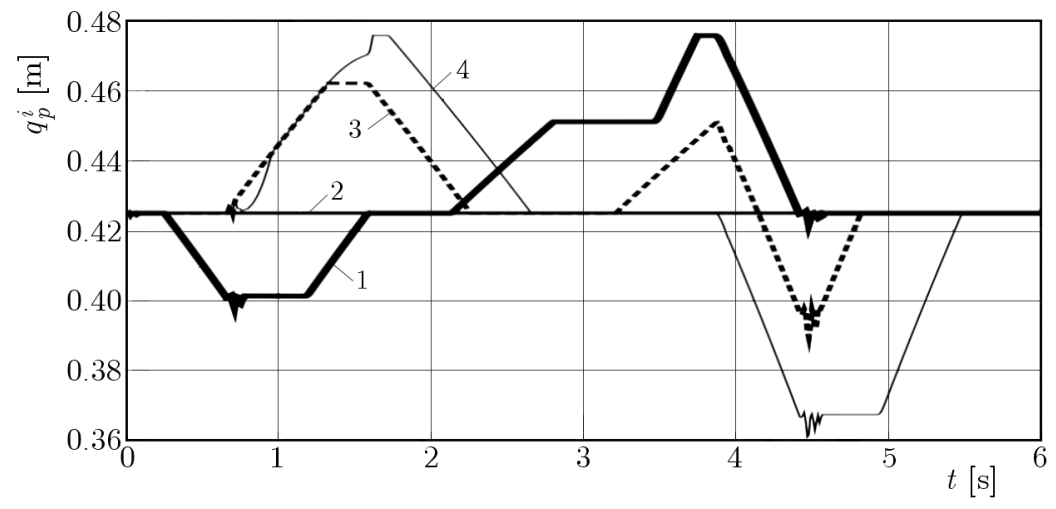

Fig. 17. Extension $q_{p}^{i}$ of the lifting actuators $(i=1,2,3,4)$

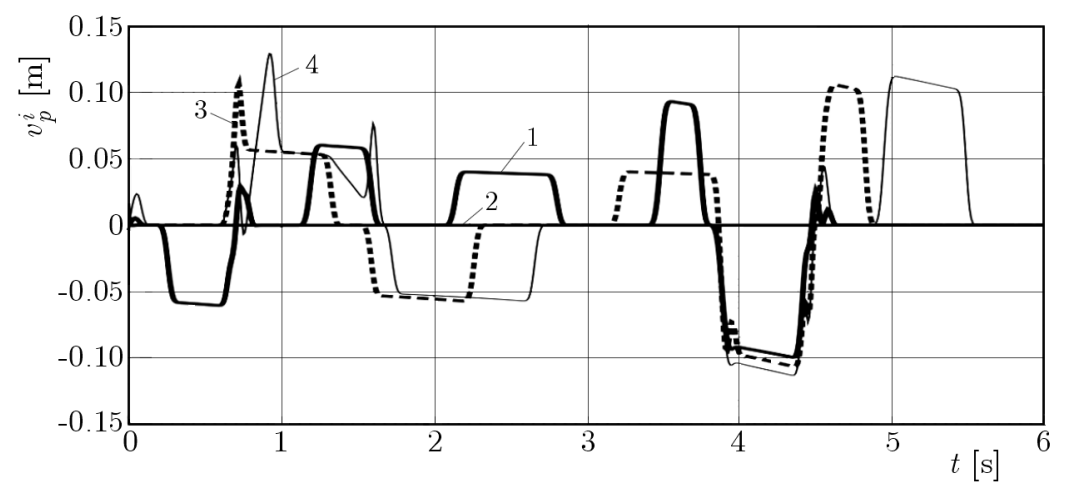

Fig. 18. Velocity $v_{p}^{i}$ of the lifting actuators $(i=1,2,3,4)$

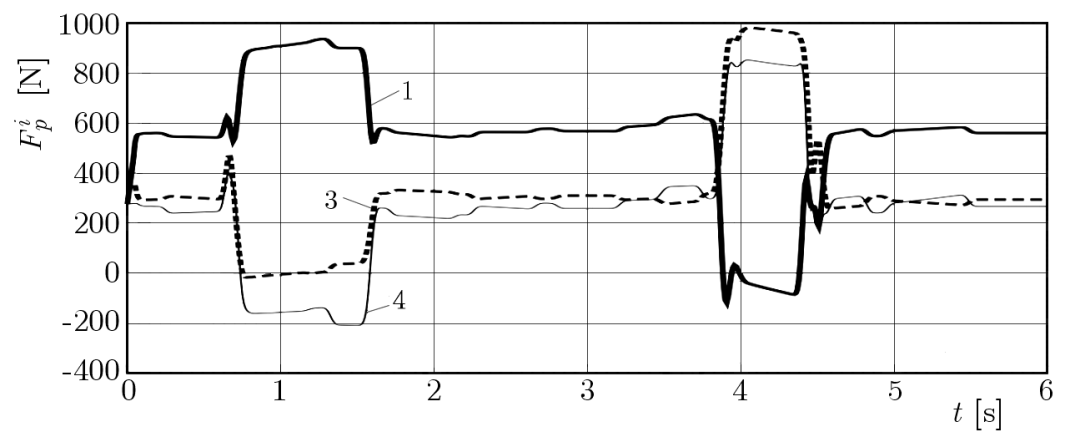

Fig. 19. Active forces $F_{p}^{i}$ in the actuators $q_{p}(i=1,3,4)$ determined by the control system

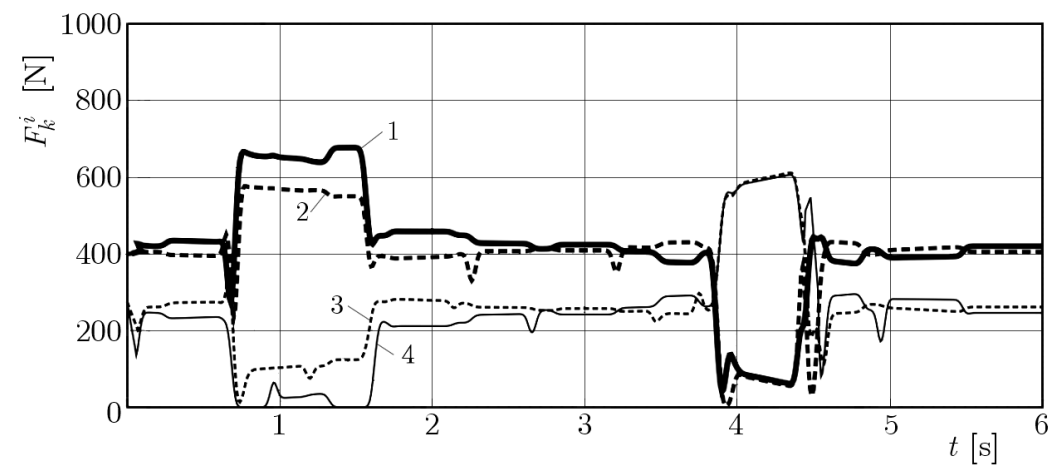

Fig. 20. Wheel-base interaction forces $F_{k}^{i}(i=1,2,3,4)$ 
whose dynamics would ensure that the required operating parameters can be exceeded is the guarantee for correct operation of the robot levelling system. As it appears from Table 2, the electric drives LINAK LA36 adopted for lifting and ejecting meet dynamic requirements since the driving forces $F_{p}^{i}$ (Fig. 19) do not exceed the nominal forces specified by the manufacturer, even during overcomming of extreme obstacles.

\section{Final remarks}

Dynamic and kinematic parameters of a wheel-legged mobile robot have been determined as a result of simulation studies. For that purpose, a numerical model of the robot and a model of the levelling control system in a computer system have been built. Robots of this kind are subject to considerable loads generated during travel on a bumpy surface. In order to build an efficient and reliable suspension system, one needs to identify the state of loading of the robot. The research has concentrated on the modelling of the leveling control system maintaining a constant orientation of the robot chassis during travel on an uneven terrain.

The analysis of the levelling system has revealed that its performance mainly depends on height of the obstacle and robot travelling velocity. The simulations validated the structure of the control system adopted for the levelling of the robot chassis and confirmed the controller parameter values to be correct. The numerical results have been used to design and build a wheel-legged robot.

\section{References}

1. BAŁChAnOwsKi J., 2012, Mobile wheel-legged robot: researching of suspension leveling system, Advances in Mechanisms Design, J. Beran (Edit.), Dordrecht, Springer

2. Batchanowski J., Gronowicz A., 2012a, Design and simulations of wheel-legged mobile robot, Acta Mechanica et Automatica, 6, 2

3. Batchanowski J., Gronowicz A., 2012b, Designing, building and dynamic studies of the autonomous wheel-legged robot (in Polish), The Project No. N N502 271037, funded by Polish Ministry of Science and Higher Education in the years 2009-2012, SPR 24/2012, Wrocław

4. Batchanowski J., Gronowicz A., 2012c, Designing and simulation examinations of a wheel-legged suspension of the wheel (in Polish), Journal of Transdisciplinary Systems Science, 16, 2

5. Gronowicz A., Szrek J., 2009a, Design of LegVan wheel-legged robot's mechanical and control system, [In:] SYROM 2009, Ion Visa (Edit.), Springer Netherlands, 145-158

6. Gronowicz A., Szrek J., 2009b, Idea of a quadruped wheel-legged robot, Archive of Mechanical Engineering, 56, 3, 263-278

7. Gronowicz A., Szrek J., Wudarczyk S., 2012, A rig for testing the leg of a wheel-legged robot, Acta Mechanica et Automatica, 6, 10

8. Halme A., Leppänen I., SuOmela J., YlÖNEn S., 2003, WorkPartner: interactive human-like service robot for outdoor applications, The International Journal of Robotics Research, 22, 7-8

9. Haug E.J., 1989, Computer Aided Kinematics and Dynamics of Mechanical Systems, Allyn and Bacon, Boston

10. HoŁdanowicz G., 2008, PIAP Robots eyes combat role, Jane's International Defence Review, July

11. http://www.bostondynamics.com/robot_petman.html

12. http://www.elbitsystems.com/elbitmain/areain2.asp?parent $=3 \&$ num $=31 \& n u m 2=31$ 
13. Sperzyński P., Szrek J. Gronowicz A., 2010, The geometric synthesis of a mechanism for realization of a rectilinear trajectory (in Polish), Acta Mechanica et Automatica, 4, 2

14. Szrek J., Wóstowicz P., 2010, Idea of wheel-legged robot and its control system design, Bulletin of the Polish Academy of Sciences. Technical Sciences, 58, 1, 43-50

15. Thhoń K., Mazur A., Dulęba I., Hossa R., Muszyński R., 2000, Manipulators and Mobile Robots (in Polish), Akademicka Oficyna Wydawnicza, Warszawa

16. Trojnacki M., Szynkarczyk P., Andrzejuk A., 2008, Tendencje rozwoju mobilnych robotów lądowych, Pomiary Automatyka Robotyka, 6/08

17. ZielińskA T., 2003, Walkingmachine (in Polish), PWN, Warszawa

Manuscript received August 5, 2014; accepted for print July 15, 2015 